\title{
Antropolog
}

\section{Los significados de la paternidad para los hombres jóvenes en los alrededores de São Paulo-Brasil}

\section{The meanings of fatherhood for young men in the surroundings of Sao Paulo-Brazil}

\section{O significado da paternidade para homens jovens na proximidade de São Paulo-Brasil}

\author{
Edemilson Antunes de Campos ${ }^{1}$, Lucas Pereira de $\mathrm{Melo}^{2}$, Danyelle Ferreira Farias ${ }^{3}$ \\ ${ }^{1}$ PhD en Ciencias Sociales en la Universidad Federal de Sao Carlos, Brasil. Profesor Doctor de la Escuela de Artes, Ciencias y \\ Humanidades. Universidad de Sao Paulo, Brasil (EACH/USP). \\ ${ }^{2}$ Doctorando del Programa Interunidades de Doctoramiento en Enfermería entre los campi de São Paulo y Ribeirão Preto de la \\ USP. Becario de Doctorado del Consejo Nacional de Desarrollo Científico y Tecnológico (CNPq). \\ ${ }^{3}$ Licenciada en Obstetricia en la Escuela de Artes, Ciencias y Humanidades de la USP.
}

Cómo citar este artículo en edición digital: Antunes de Campos E; Pereira de Melo L; Ferreira Farias D. (2012) Los significados de la paternidad para los hombres jóvenes en los alrededores de São Paulo-Brasil. Cultura de los Cuidados. (Edición digital) 16, 33. Disponible en: <http://dx.doi.org/10.7184/cuid.2012.33.08>

Correspondencia: Edemilson Antunes de Campos. Escola de Artes, Ciências e Humanidades - Universidade de São Paulo. Rua Arlindo Bettio, 1000, Ermelino Matarazzo. São Paulo-SP, Brasil, CEP: 01238-000/

Correo Electrónico: edicampos@usp.br/Teléfono: 5511 5245-2602

Recibido: 06/11/2011/Aceptado: 24/marzo/2012

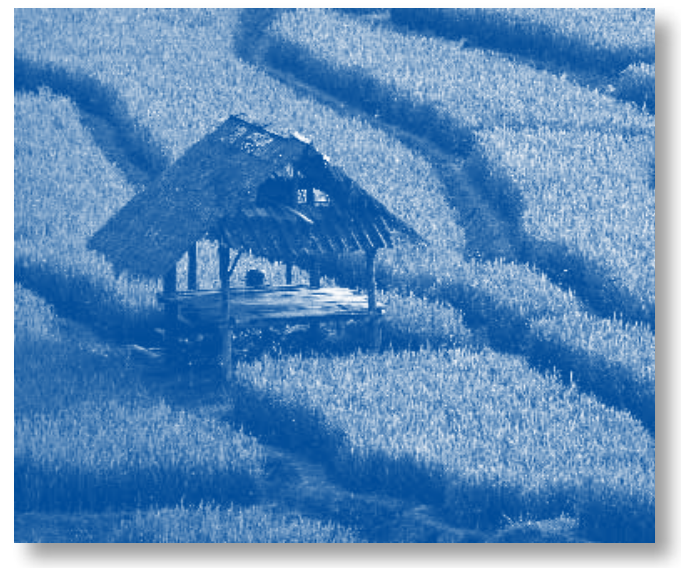

\section{ABSTRACT}

The aim of this study was to understand the representations and meanings about the fatherhood of young men, through analysis the social codes of the sociocultural context in which they are inserted. This is a qualitative research. The young men were interviewed. Thematic analysis was conducted and data were analyzed by sentences. The meanings of fatherhood are tied to the representations and to the practices built on the body, the sexuality and the reproduction inside a logic that operates with the own values of the family and work of the sociocultural context. It is looked, so, to make the existent repertoire rich on the understanding of the senses of the fatherhood between young men and, for this road, to contribute to the establishment of public policies that effectively dialogue and that bases on the logic that orientates his practices around the sexual and reproductive life.

Key-words: Gender. Pregnancy in Adolescence. Fatherhood. Social Values. Cultural, Anthropology. 


\section{RESUMO}

O objetivo desta pesquisa é compreender as representações e os significados sobre a paternidade de homens jovens, por meio dos códigos sociais próprios ao contexto sociocultural no qual eles estão inseridos. Trata-se de uma pesquisa qualitativa na qual realizaram-se entrevistas semi-estruturadas com homens jovens da periferia da cidade de São Paulo, Brasil. Os sentidos da paternidade estão ligados às representações e às práticas construídas sobre o corpo, a sexualidade e a reprodução dentro de uma lógica que opera com os valores da família e do trabalho próprios ao contexto sociocultural no qual eles estão inseridos. Busca-se, assim, enriquecer o repertório existente sobre a compreensão dos sentidos da paternidade entre jovens e, por essa via, contribuir para o estabelecimento de políticas públicas que efetivamente dialogue e se baseie na lógica que orienta suas práticas em torno da vida sexual e reprodutiva.

Palavras-chave: Gênero. Gravidez na Adolescência. Paternidade. Valores Sociais. Antropologia Cultural.

\section{RESUMEN}

El objetivo de esta investigación es comprender los significados y representaciones sobre la paternidad de los hombres jóvenes, a través de los códigos sociales propios del contexto sociocultural en el que están. Se trata de una investigación cualitativa en que se realizaron entrevistas semi-estructuradas con los hombres jóvenes en los alrededores de São Paulo, Brasil. El significado de la paternidad está vinculado a las representaciones y las prácticas establecidas sobre el cuerpo, la sexualidad y la reproducción dentro de una lógica que opera con los valores de la familia y del trabajo, propios del contexto sociocultural estudiado. El objetivo es enriquecer el repertorio existente sobre el entendimiento actual de los significados de la paternidad entre los hombres jóvenes, y de esta manera, contribuir al establecimiento de políticas públicas que efectivamente discutan y tengan como cuenta la lógica que guía sus prácticas en la vida sexual y reproductiva.

Palabras clave: Género. El embarazo en la adolescencia. Paternidad. Valores sociales. Antropología Cultural.

\section{INTRODUCCIÓN}

La vida sexual y reproductiva de los hombres jóvenes ha generado debates importantes en la sociedad brasileña. Un ejemplo es el "embarazo en la adolescencia", considerado uno de los temas más importantes de salud pública actualmente, llamando la atención de investigadores y profesionales de la salud, educación, justicia y de las ciencias sociales, entre otros.

En este contexto, las políticas públicas y los debates académicos se han centrado en la aplicación de programas de planificación, gestión y promoción de la salud centrados en la salud materna e infantil, incluyendo la salud de las madres adolescentes. Sin embargo, se observa en la literatura, una incipiente discusión y falta de acciones estratégicas dirigidas a la salud de los hombres adolescentes (Trivedi, Brooks, Bunn, \& Graham, 2009; Lohan, Crucero, O'Halloran, Alderdice, \& Hyde, 2010).

En Brasil, estos aspectos se han reflejado también en el tema de la salud sexual y reproductiva de los adolescentes. Los enfoques que han prevalecido en algunas investigaciones sobre la sexualidad en la adolescencia son los que favorecen las experiencias de las mujeres (Lyra, 1997). 
El abandono de la perspectiva de hombres adolescentes, tanto en las investigaciones como en las políticas sociales y de salud ha producido impactos negativos en la experiencia de paternidad de estas personas. Lohan, Cruise, O'Halloran, Alderdice, \& Hyde (2010) destacan que el embarazo en la adolescencia no debe ser visto simplemente como una cuestión de la mujer. Sostienen además que la comprensión de la perspectiva de los adolescentes puede llevar a medidas para prevenir el embarazo y a programas de asesoramiento más eficaces.

Los estudios también muestran que, actualmente, las acciones de promoción de la salud sexual de este grupo de población son inadecuadas para responder a sus necesidades reales. También se argumenta que los jóvenes tenían ideales similares a las jóvenes. Sin embargo, los programas de salud existentes son problemáticos porque destacan los estereotipos negativos de los hombres jóvenes, de los modelos de masculinidad o de las dificultades para mantener una relación significativa (Trivedi, Brooks, Bunn, \& Graham, 2009).

En este sentido, el embarazo en la adolescencia suele tratarse con un enfoque universal y normativo, que ignora los aspectos sociales y culturales que dan forma al contexto en el que los hombres jóvenes están incluidos.

En el contexto brasileño, la crítica de este enfoque ha ganado impulso, especialmente a través de las investigaciones que se centran en las relaciones de género en el campo de la sexualidad y salud reproductiva (Suárez et al., 1999; Barbosa et al., 2002; Heilborn, 2006; Villela, Arilha, 2003; Scavone, 2004; Correa, 2005; Aquino et al., 2006).

Los estudios de las relaciones de género son importantes en la comprensión de la vida sexual y reproductiva de hombres y mujeres, contribuyendo tanto para destacar la discipli- na y la normalización dirigidas a las mujeres, como para haber espacio para el estudio de las masculinidades, que apuntan la necesidad de incluir a los hombres en las políticas públicas de salud sexual y reproductiva (Arilha, 1999; Nascimento, 1999; Medrado et al., 2000; Medrado, Lyra, 2002; Aquino et al., 2006; Barker, 2008; Scott, 2010).

Así, el objetivo de este trabajo es entender las representaciones y significados de la paternidad entre los hombres jóvenes que viven en los alrededores de Sao Paulo, Brasil, y de esta manera, comprender la lógica que guía sus vidas sexual y reproductiva. El ejercicio de la sexualidad y la vida reproductiva requiere un aprendizaje dentro de una lógica, que se rige por las relaciones de género, fortaleciendo los códigos sociales y culturales que dan forma al contexto social y cultural en el que los jóvenes están incluidos.

Se parte del principio de que los significados de la paternidad entre los hombres jóvenes están vinculados a las representaciones y las prácticas establecidas sobre el cuerpo, la sexualidad y la reproducción dentro de una lógica que opera sus propios valores en el contexto sociocultural en el que se incluyen, especialmente los valores de "familia" y "trabajo".

Las representaciones sociales aquí son entendidas como "una forma de conocimiento, socialmente elaborado y compartido con un objetivo práctico, y que contribuye a la construcción de una realidad en un grupo social" (Jodelet, 2001, p. 22).

Las representaciones componen los sistemas de interpretación social que permiten a los individuos formular una comprensión de la experiencia, guiando a sus prácticas sociales. A los jóvenes padres entrevistados, cuando se habla de la paternidad, movilizan un conjunto de representaciones fundamentales para 
la comprensión de los significados de ser padre en la adolescencia y sus responsabilidades dentro de la familia y del trabajo.

Se pretende enriquecer el repertorio existente sobre el entendimiento actual de los significados de la paternidad entre los hombres jóvenes, y de esta manera, contribuir al establecimiento de políticas públicas que efectivamente discutan y tengan como base la lógica que guía sus prácticas en la vida sexual y reproductiva.

\section{METODOLOGÍA: CONOCIENDO Y HABLANDO CON PADRES JÓVENES}

Se trata de una investigación cualitativa de carácter antropológico, ya que se trabajó "con el universo de significados, motivaciones, aspiraciones, creencias, valores y actitudes, lo que corresponde a un espacio más profundo de las relaciones, procesos y fenómenos que no se pueden reducir a la operación de variables" (Minayo, 1994, p. 22).

El trabajo de campo se realizó en São Paulo, sudeste de Brasil, de abril a agosto de 2008. La ciudad de São Paulo se sub-divide en 09 regiones de acuerdo con la ubicación geográfica

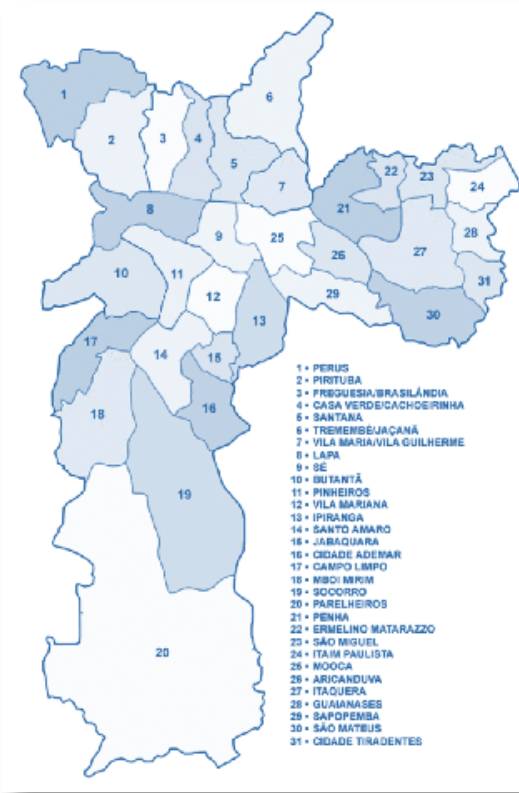

Figura 01 - Distribución geográfica de las Subprefecturas de la Ciudad de São Paulo, Brasil y características históricas

y culturales. Estas regiones son: Central, Noroeste, Norte o Nordeste, Este 1 y 2, Sureste, Sur, Centro-Sur y Oeste. Además de la subdivisión regional, la ciudad se organiza administrativamente en 31 Sub-prefecturas (Figura 01).
El escenario cultural investigado fue el barrio Jardín Keralux, ubicado en la Sub-prefectura de Ermelino Matarazzo, zona este de la ciudad. El Jardín Keralux es el resultado de un proceso de urbanización irregular ocurrido en 1996. Es un barrio que se concentra principalmente una población trabajadora, que depende de su salario para su reproducción social. En este barrio el paisaje urbano se compone de casas sencillas, sin terminar o en construcción. Teniendo en cuenta los arreglos variados de la estructura familiar, estas casas sirven como lugar de residencia a más de una familia al mismo tiempo (los propietarios y sus hijos cuando se casan).

$\mathrm{O}$ acceso a los informantes se obtuvo a través de la mediación de la Unidad Básica de Salud local (UBS). El encuentro con los jóvenes padres no fue una tarea fácil. La investigación implicó en viajes y (des)encuentros que constituyen el núcleo de trabajo del investigador. El trabajo de investigación social consiste en entrar en el mundo de otro en un contacto estrecho entre investigador e investigado.

Durante el trabajo de campo se percibió que hubo resistencia por parte de la población local cuando se hablaba sobre entrevistas y en grabarlas. La gente tenía miedo de hablar sobre sus vidas, en particular su vida sexual. Esta actitud continuó mismo después de la explicación de los objetivos de la investigación y la presentación del formulario de 
consentimiento, para garantizar que las entrevistas serían confidenciales y que sus nombres no serían revelados.

Con la presencia de los investigadores y una relación más estrecha con los informantes, fue posible localizar a algunos padres jóvenes para participar del estudio. Esta "vía", fue obtenida durante una conversación informal con uno de los profesionales de la UBS local, que nos dio las direcciones y los datos de 09 padres jóvenes. Hicimos contacto personal con estos jóvenes y presentamos los objetivos de la investigación y como participarían. Tres hombres jóvenes aceptaron participar.

Los jóvenes que participaron de la investigación tienen las siguientes características:

- Almir, 24 años, vive en unión estable, educación primaria incompleta, trabajador de la construcción desempleado y padre de un hijo que nació cuando tenía 17 años;

- Ademar, 24 años, vive en unión estable, educación primaria incompleta, trabajador de la construcción desempleado y padre de tres hijos, y tuvo su primer hijo a los 16 años;

- Richard, 20 años, vive en unión estable, educación secundaria incompleta, guardia de seguridad desempleado, padre de dos niños, tuvo su primer hijo a los 17 años.

Para colectar las informaciones fueran realizadas entrevista semi-estructurada. Las entrevistas con los jóvenes padres fueron hechas en días, horas y lugares escogidos de acuerdo con la disponibilidad de los jóvenes y duró entre 40 minutos y una hora. Se les informó de los objetivos de la investigación y firmaron un formulario de consentimiento.

Las entrevistas fueron transcritas e hicieron análisis temáticos, destacando las representaciones y significados sobre la paternidad de los hombres jóvenes y su relación con los valores que configuran el contexto sociocultural en el que están, en particular la familia y el trabajo.

El proyecto de investigación fue aprobado por el Comité de Ética de la Escuela de Enfermería de la Universidad de São Paulo (CEP/ EEUSP) bajo proceso de número 752/2008.

\section{LOS SIGNIFICADOS DE LA PATERNI- DAD DE JOVENES DEL ALREDEDOR DE SÃO PAULO}

Los resultados y la discusión están organizados en categorías:

I) "EElla iba a vivir con un hombre, no con un muchacho!": la familia y la paternidad.

II) "Cuando un hombre asume una familia tiene que ser responsable": el trabajo y la paternidad.

III) Las representaciones y significados de la paternidad y el embarazo en la adolescencia para los hombres jóvenes.

\section{“Ella iba a vivir con un hombre, no con un muchacho!": la familia y la paternidad}

Los relatos de los hombres jóvenes destacan la importancia del ámbito de la familia en su experiencia de paternidad. La literatura antropológica ha demostrado el papel central que ocupa la familia en las relaciones sociales y en la definición de la identidad social, especialmente entre los miembros de las clases bajas.

Zaluar (1994) en su estudio etnográfico enfatiza el valor de la identidad familiar y social de "trabajador" para los residentes de los barrios pobres. Para la autora, "el trabajador es miembro de una familia respetable, cuya sobrevivencia se da aumentando los ingresos familiares. El respeto que tiene por sí mismo y para con otros proviene de allí. Esta es la obligación de los miembros de la familia, sobre 
todo cuando se es el principal proveedor, se acepta como positivo el trabajo" (Zaluar, 1994, p. 89).

En este sentido, la familia no se puede reducir a una invención burguesa, sino que incluye una constelación de derechos y deberes, creando enlaces recíprocos y vínculos entre sus miembros, lo que hace a la familia un centro clave en la irradiación de los valores que definen la identidad y la noción de persona entre los miembros de las clases bajas.

Sarti (2005a; 2005b) también ofrece una importante contribución con su investigación sobre la importancia de la esfera familiar en la definición, al mismo tiempo, de un orden moral y un universo simbólico de los habitantes de los alrededores de São Paulo. Para esta investigadora, la familia funciona como un centro de referencia simbólica en los medios populares, de manera que la idea del espacio familiar "como un orden moral, es el espejo que refleja la imagen con la cual los pobres ordenan y dan sentido al mundo social" (Sarti, 2005b, p. 22).

El universo simbólico de la familia, con sus códigos de reciprocidad y obligaciones, es una parte integral de la compleja formación histórica de la sociedad brasileña, lo que permite a los "pobres" un principio organizador de su percepción del mundo (Sarti, 2005b).

La familia, con sus códigos y obligaciones, se considera como un lenguaje que articula los elementos de la vida social, lo que permite a sus miembros dar un significado a la vida, mientras que definen los contornos de su identidad social.

Desde esta perspectiva, la familia es más de un núcleo de la sobrevivencia material y espiritual de sus miembros, es el sustrato "de su identidad social [y] su importancia no es funcional, su valor no es meramente instrumental, sino que se refiere a su identidad de ser

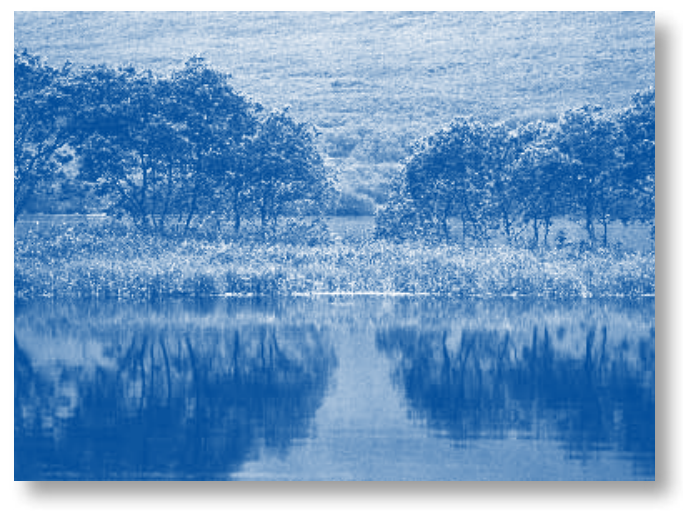

social y constituye la referencia simbólica que estructura su explicación del mundo" (Sarti, 2005b, p. 53).

Los estudios demográficos han destacado la importancia de la familia como un "factor de protección social” en los países de América Latina, especialmente en Brasil, debido a los cambios causados en el orden económico en las últimas décadas: "la centralidad de la familia como un factor de protección social requiere tener en cuenta su carácter activo y participante en los procesos de cambio en curso $y$, al mismo tiempo, los cambios internos, en particular en su dimensiones de la sexualidad, la procreación y el compañerismo" (Goldani, 1999, p. 41).

En medio de todo este proceso de transformación socioeconómica "las familias permanecen en el centro de la procreación y socialización de las nuevas generaciones y, por tanto, adquieren una importancia fundamental como un espacio transmisor de oportunidades y las perspectivas de vida de sus miembros (...) que hace con que la familia persista y se reproduzca en una especie de espacio de 'conflicto cooperativo"' (Goldani, 1999, p. 43-4).

En la sociedad brasileña, con la inestabilidad y la inseguridad generada por la exclusión social, "la familia tiende a ser en un punto fijo alrededor del cual las identidades se estructuran e los trabajadores y trabajadoras puede 
contar una historia y construir un biografía, dar coherencia a los acontecimientos vividos, asignar un significado a sus experiencias $y$ construir proyectos de futuro, haciendo su mundo muy difícil de vivir (...) en una zona de 'conflicto cooperativo', en que se cruzan las diferencias por género y entre las generaciones" (Goldani, 1999, p. 65).

Los datos de este estudio apoyan estas discusiones acerca de la familia. Richard estaba seguro de regresar a casa con sus padres, después de haber intentado vivir solo:

"Hubo una pequeña confusión y tuvimos que ir a casa de mis padres. Porqué no conseguía pagar el alquiler, poner comida en la casa y las medicinas para los niños. Desde entonces he estado viviendo en la casa de mi madre. Estoy bien, está todo bien."

Este discurso muestra que los padres de los jóvenes es muy importante en esta decisión, en la medida que asumen junto con los hijos la responsabilidad, aceptando la nueva familia en su propio hogar, y contribuyen al sostenimiento y en los cuidados con los niños (Correa, 2005).

A veces, la familia actúa como un espacio de conflicto, ya que no apoya el embarazo por lo considerar prematuro y no deseado, y tener que asumir la responsabilidad del cuidado del niño o por no aprobar su pareja.

Para Ademar, las dos uniones que llevaron a sus hijos fueran confusas y por las dificultades impuestas por las familias de sus compañeras, tuvo que quedarse lejos de sus hijos y de su creación:

"Hubo una pelea allá [en la casa de la familia de ella] porque su padre empezó a golpear a su madre. Tuve que entrar en la pelea. Yo hablé así: 'iNo, de esa manera no es posible! ¡No va a funcionar, porque no voy a aceptar!'. Luego conocí a otra mujer y me quedé con ella. Pero fue la misma cosa: ¡de ser mandado por sus hermanos! ¡A mí no me gusta! Pasamos unos tiempos juntos y nos separamos. Luego conocí a mi actual esposa y estamos juntos hasta hoy."

Para Almir, tomar la novia de su casa y traerla a vivir con él, fue uno de los momentos en que tuvo que enfrentar la familia y asumir la responsabilidad de ser "jefe de familia":

"Llegué a la casa de su tía y le dije que la estaba llevando conmigo, ella se quedaría conmigo. Su tía me dijo que si ella fuera conmigo no la aceptaría de nuevo. ;Le dije que iba a vivir con un hombre, no con un muchacho! Y estamos juntos hasta hoy."

Este relato refuerza la idea presentada por Duarte (1986), que apunta la centralidad que la familia ocupa entre los miembros de las clases más bajas, operando con un valor en la construcción de la identidad social y de la noción de persona en su interior. Para este autor, "el valor de la familia abarca una serie de cualidades distribuidas entre sus componentes y que le dan su preeminencia como foco de la identidad social." (Duarte, 1986, p. 175).

Así, asumir la responsabilidad de una familia es uno de los vectores que definen la identidad de los hombres jóvenes que vienen a se reconocen como padres.

\section{"Cuando un hombre asume una familia tiene que ser responsable": el trabajo y la paterni- dad}

Un tema recurrente también se refiere a la cuestión de la responsabilidad por el proveimiento de la familia a través del trabajo. Convertirse en un hombre se correlaciona directamente con la responsabilidad de proveer para su familia (esposa e hijos) a través del trabajo. En un estudio hecho con jóvenes padres de- 
tenidos, en Escocia, Buston (2010) dice que todos tienen el deseo de ser "un buen padre." Además, los jóvenes padres entrevistados no se sienten preparados para ser padres, sobre todo porque se sienten incapaces de cumplir el rol de proveedor de servicios financieros.

Sarti (2005b) señala la importancia del trabajo con sentido de "valor" entre los hombres, "en la moral del hombre, ser un hombre fuerte para trabajar es una condición necesaria, pero no suficiente para la afirmación de su virilidad. Un hombre, para ser hombre, también necesita una familia “(Sarti, 2005b, p. 95 - Énfasis del original).

Eso es lo que Ademar dice que tiene "orgullo" de ser un trabajador: "Yo siempre he trabajado, tengo todos mis defectos, pero trabajar, yo trabajo y soy orgulloso de esto". O también:

"Yo nací llevando y recogiendo naranjas, agitando maní, tomates, guayabas. ¡Allí donde yo vivía no tenía esa libertad de servicio que tenemos aquí! ¡Era sólo el campo! Siempre he trabajado: servicios con maderas... Lo que surge, lo hago, ¿sabes? Para mí no hay ninguna excepción."

El hombre necesita tener la condición de “jefe de familia”, es decir, la condición de aquel que tiene una obligación moral de proveer la familia a través de su trabajo. Por lo tanto, la "moral del hombre fuerte" gira en torno a la "moral del proveedor", una manera particular de definir los contornos de la identidad de los padres jóvenes, definidos como aquellos que son responsables tanto por el cuidado de sí mismo como proveer su familia.

Además, cuanto al cuidado de sí mismos, cuando la experiencia del hombre é marcada por la participación en el proceso de confirmación del diagnóstico de embarazo, ayúdale en la transición a la paternidad temprana (Draper, 2002).
Eso es lo que dice Almir, cuando relata su reacción al enterarse del embarazo de su novia:

"Cuando ella dijo que estaba embarazada fue una bendición de Dios! Llegó llorando. Le dije: ‘Por qué lloras?’ Y ella me dijo: 'Puede que no quieras'. Yo dije: ¿Desde cuando te asumí, acepto lo que venga y lo que deje de venir!' Creo que cuando un hombre asume a una familia tiene que ser responsable de todo. Gracias a Dios, hoy lo soy."

El valor del trabajo también puede ser entendido como una base de la autonomía. Según dice Sarti (2005b), el trabajo más allá de su valor económico abarca un sentido moral y es el fundamento de la autonomía del hombre. En este sentido, el trabajo también está relacionado con la salud y la sanidad del cuerpo como un instrumento que lo pone en el mundo del trabajo.

Eso es lo que se deduce de la narración de Richard, cuando señala la importancia del trabajo para no depender de sus padres:

"Trabajé dos años en esa empresa porque hubo una pequeña confusión y me despidieron. Desde entonces estoy sudando la camisa para conseguir un trabajo (...) porque no quiero depender de mi padre y mi madre para nada."

Sarti (2005b) destaca una especie de equivalencia entre una "ética del proveedor" y una "ética de trabajo" de modo que, es en el entrelazamiento entre las lógica que rigen estas dos éticas que se construye la identidad del hombre de las clases bajas. Es decir, las relaciones de trabajo articulan factores económicos a los elementos morales de la actividad laboral.

La lógica que rige el trabajo para los hombres jóvenes entrevistados también parece obedecer a una regla que destaca los valores morales de "hombre proveedor". La concepción de trabajo está relacionada con el valor de 
la paternidad en una trama en la cual se reconocen como hombres responsables de proveer a su familia.

Es así que se construyen los significados de la paternidad y del embarazo en la adolescencia para los hombres jóvenes, tejiendo una conexión entre los valores morales de la "familia" y del "trabajo" que guía sus acciones.

\section{Representaciones y significados de la paterni-} dad y del embarazo en la adolescencia para los hombres jóvenes

La paternidad para padres jóvenes significa la confirmación de su masculinidad. En general, sólo se sienten responsables cuando los hombres tienen que asumir las consecuencias de sus acciones.

Aunque se le considera como un evento inesperado, el embarazo también plantea la cuestión de la responsabilidad de los hombres jóvenes, como también mostró Buston (2010). Eso es lo que dice Almir, cuando relata su reacción al enterarse del embarazo de su novia:

"No me ocurrió tener hijos. Pero sucedió. No tuve manera, ¿sabes? Pero querer, yo no quería. Gracias a Dios, hoy lo soy."

O en las palabras de Richard, que, cuando cobrado por sus padres, les dije que cuidará de su hija y su compañera, asumiendo su responsabilidad. En sus palabras:

"Cuando se quedó embarazada, mi padre y mi madre no lo sabían. Sólo supieron después de los 8 meses. ¡Mis padres se quedaron "atónitos", con "la boca abierta"! Ellos nunca pensaron que yo iba a tener dos hijas ahora. Me preguntaron cómo iba a hacer para darles de comer. Yo dijo: ‘ A mi manera! Las cosas que quiero yo consigo"'.

El embarazo aparece para Almir como una "bendición de Dios", mostrando cómo este evento es visto como una "fatalidad", mediada por la intervención divina. Frente a esta situación sin más remedio, tuve que aceptarla.

La cuestión de la responsabilidad en la paternidad también parece asociada con el uso o no de métodos anticonceptivos. Los jóvenes padres revelan que sabían las formas de evitar un embarazo no deseado, pero no hizo nada para prevenir.

"Cuando nos conocimos, teníamos conocimiento del embarazo, pero no evitamos. Ella quedó embarazada por accidente, ¡sin intención! No pudimos evitar" (Richard).

$\mathrm{O}$, en el discurso de Almir:

"No nos preveníamos. Siempre he dicho que si teníamos un hijo sería bueno lo suficientemente. ;Yo no me preocupaba en usar nada! Yo le digo que se quede tranquila, 'Si se queda embarazada una vez más será de la misma manera: de la forma en que tuvimos el primer, tenemos el segundo, el tercero...' Es costumbre nuestro, no hay ninguna razón para evitar."

Cabral (2003) sugiere que la ocurrencia de embarazo se asocia con los tipos de relaciones que los hombres jóvenes tienen con sus parejas, y no precisamente con la falta de información sobre anticonceptivos. Con sus novias y "las mujeres de la casa" no habría necesidad de utilizar un método anticonceptivo, ya que en ellas se "puede" confiar. Para estos jóvenes el uso o no de condón está relacionado con la afectividad de la relación, no con la preocupación de prevenir enfermedades o un embarazo no deseado.

Buston (2010), en su estudio, afirma que algunos adolescentes ven la posibilidad de que la pareja se queda embarazada como algo remoto. Por eso no pensaban en el asunto o pensaban poco. Sin embargo, entre la minoría que 
informó pensar en la posibilidad de embarazo, el uso de anticonceptivos fue alta.

Ser padre para estos jóvenes significa también una entrada en la edad adulta, dando lugar a una nueva forma de tomar la vida a través de la responsabilidad de la paternidad. Eso es lo que Richard señaló al referirse a las transformaciones sufridas desde que es padre:

"Para mí, mucho ha cambiado: yo casi no me quedaba en casa, salía para bares. Esto de ser padre, para mí, ¡cambió muchas cosas! ¡Hoy la paternidad es vivir con mi hijo! Educar y enseñar el día a día."

Estos datos corroboran los resultados de Mollborn \& Lovegrove (2011). Para los autores, las influencias ejercidas por los padres adolescentes en sus hijos es diferente de las que ejercen los padres adultos con respecto al contexto familiar, la relación entre los padres; y la relación padre-hijos. El estudio mostró que el estado civil de los padres y el contexto familiar tienen un impacto en el desarrollo cognitivo y en el comportamiento de los niños (Mollborn \& Lovegrove, 2011). La representación de la paternidad que implica en vivir con su hijo, señalada por Richard, además de su valor moral trae consecuencias positivas para el crecimiento y desarrollo de sus hijos.

Pero para Almir y Ademar, la paternidad ha cambiado sus vidas, pues dejaron de hacer cosas de "soltero", de actuar de manera irresponsable $\mathrm{y}$, de alguna manera, se sienten más maduros:

"Cuando estaba soltero pensaba en muchas cosas diferentes. ¡Una vez que se tiene una familia 'yo levanto la mano' $y$ miro lo que tengo dentro de casa antes de hacer algunas cosas malas que hiciera cuando estaba solo! Tengo que agradecer a Dios por tener una familia en casa $y$ dar más valor para ellos. ¡Ser padre fue algo muy bueno!"
Esta representación del padre joven como un "hombre responsable" confirma las conclusiones de la investigación hecha por Cabral (2003), realizada con hombres jóvenes que viven en barrios marginales de la ciudad de Rio de Janeiro, en que también afirma los detalles de las representaciones y prácticas de estos jóvenes acerca de su vida sexual y reproductiva, y de esta manera, sus relaciones con la experiencia de la paternidad.

Para esta autora, "el nacimiento y asunción de un hijo parecen facilitar la transición a la edad adulta. 'Asumir la responsabilidad', una consecuencia directa de la paternidad, implica la incorporación o la asunción de nuevos roles caracterizados por los atributos de 'seriedad' y 'madurez"' (Cabral, 2003, p. 290).

Así, la paternidad significa una transición que inicia los hombres jóvenes en la edad adulta, dentro de un contexto en el que la responsabilidad por el proveimiento de la familia significa, esencialmente, se convierte en un hombre. También de acuerdo con Cabral (2003, p. 289): "los jóvenes parecen decir que 'se convierten en hombres' asumiendo la paternidad, $o$ antes, que es necesario 'ser hombre' para 'tener responsabilidades' y asumir lo que hizo."

Este enfoque de la juventud como un proceso tiene un valor heurístico, lo que permite cuestionar las generalizaciones sobre el desarrollo de los jóvenes, ya que revela la importancia de los aspectos socioculturales que permean las relaciones entre las clases sociales en la definición de su biografía.

Así, mientras en las clases media y alta la transición de la adolescencia a la adultez comprende una secuencia de eventos que van desde la escuela a la universidad, dando prioridad a la inclusión en el mercado laboral, la estructura familiar, y más tarde el nacimiento de los niños; para las clases populares, esta transición 


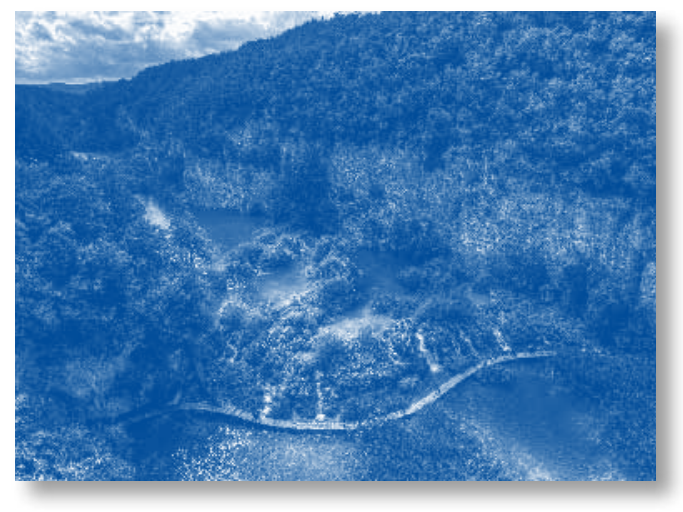

parece ser formada por los valores propios del contexto sociocultural en el que los hombres jóvenes están incluidos. Destacase, sobre todo los valores de la "familia" y del "trabajo", de manera que el embarazo puede significar "un medio de promoción social" dentro de un proyecto de autonomía, en que se convertir en "padre", esencialmente, es se convertir en "responsable" de proveer su familia.

\section{CONSIDERACIONES FINALES}

Esta investigación tuvo como objetivo comprender los significados y representaciones sobre la paternidad de los jóvenes que viven en los alrededores de São Paulo, y de esta manera, comprender la lógica que rige la vida sexual y reproductiva de estos jóvenes.

Para nuestros informantes la paternidad es un proceso de afirmación de la masculinidad, vinculada a los valores morales de "familia" y “trabajo". Ser un hombre es una construcción que moviliza a los hombres jóvenes los valores que dan forma al contexto en el que se incluyen.

La lógica que rige sus vidas sexual y reproductiva está directamente relacionada con los valores de la familia y el trabajo y se regirán por las relaciones de género que definen las relaciones asimétricas entre hombres y mujeres. Por lo tanto, el embarazo en la adolescencia es concebido como un evento que da responsa- bilidad al joven en proveer su familia. Ser un proveedor parece ser el valor que guía sus acciones para asumir la responsabilidad del cuidado de sus familias.

Y así, todos los días, los hombres jóvenes redibujan los contornos de su construcción subjetiva, dentro de una lógica regida por las relaciones de género, cuyas reglas movilizan los valores propios del contexto sociocultural en que se encuentran, sobre todo los valores de "familia "y" trabajo ".

\section{REFERENCIAS BIBLIOGRÁFICAS}

- Aquino, E.M. et al. (2006). Gravidez na adolescência: a heterogeneidade revelada. In M.L. Heilborn et al. (Eds.), O aprendizado da sexualidade: reprodução e trajetórias sociais de jovens brasileiros (pp.310-60). Rio de Janeiro: Garamond/FIOCRUZ.

- Arilha, M. (1999). Homens, saúde reprodutiva e gênero: o desafio da inclusão. In K. Giffin \& S. H. Costa (Eds.), Questões da saúde reprodutiva (pp.455-67), Rio de Janeiro: FIOCRUZ.

- Barbosa, R. M. et al. (2002). Interfaces: gênero, sexualidade e saúde reprodutiva. Campinas - SP: Editora da UNICAMP.

- Barcker, G. (2008). A saúde do homem adolescente: uma perspectiva de gênero aplicada ao masculino. In Brasil. Ministério da Saúde. Secretaria de Atenção à Saúde. Departamento de Ações Programáticas Estratégicas. Saúde do adolescente: competências e habilidades (pp.223-30). Brasília: Editora do Ministério da Saúde.

- Buston K.M. (2010). Experiences of, and attitudes towards, pregnancy and fatherhood amongst incarcerated young male offenders: findings from a qualitative study. Social Science and Medicine, 71, 2212-8.

- Cabral, C. S. (2003). Contracepção e gravidez na adolescência na perspectiva de jovens pais de uma comunidade favelada do Rio de Janeiro. Cadernos de Saúde Pública, 19 (Supl. 2), pp. 283-292.

- Corrêa, A. C. P. (2005). Paternidade na adolescência: vivencias e significados no olhar de homens que a experimentaram. Tese de Doutorado, Escola de Enfermagem, 
Universidade de São Paulo, Ribeirão Preto-SP, Brasil.

- Draper J. (2002). "It's the first scientific evidence": men's experience of pregnancy confirmation. Journal of Advanced Nursing, 39, 563-70.

- Duarte, L. F. D. (1986). Da vida nervosa nas classes trabalhadoras. Rio de Janeiro: Jorge Zahar Editor.

- Goldani, A. M. (1999). O regime demográfico brasileiro nos anos 90: desigualdade, restrições e oportunidades demográficas. In L. Galvão \& J. Díaz (Eds.), Saúde sexual e reprodutiva no Brasil (pp.40-66). São Paulo: Hucitec/ Population Council.

- Heilborn, M. L. et al. (2006). O aprendizado da sexualidade: reprodução e trajetórias sexuais de jovens brasileiros. Rio de Janeiro: Garamond/FIOCRUZ.

- Jodelet, D. (2001). As representações sociais. Rio de Janeiro: Ed. UERJ.

- Lyra, J. (1997). Paternidade adolescente: uma proposta de intervenção. Dissertação de Mestrado, Pontifícia Universidade Católica de São Paulo, São Paulo, Brasil.

- Lohan M., Cruise, S., O’Halloran, P., Alderdice, F., \& Hyde, A. (2010). Adolescent men's attitudes in relation to pregnancy and pregnancy outcomes: a systematic review of the literature from 1980-2009. Journal of Adolescent Health, 47, 327-45.

- Medrado, B. \& Lyra, J. (2002). Produzindo sentidos sobre o masculino: da hegemonia à ética da diversidade. In M. Adelman \& C. Silvestrin (Eds.), Coletânea gênero plural (pp.63-76). Curitiba: UFPR.

- Medrado, B.; Lyra, J.; Galvão, K.; Nascimento, P. (2000). Homens por quê? Uma leitura da masculinidade a partir de um enfoque de gênero. Perspectivas em Saúde e Direitos Reprodutivos, 3, pp. 12-6.

- Minayo, M.C.S. (Ed.) (1994). Pesquisa social: teoria, método e criatividade. Petrópolis: Vozes.

- Mollborn S., \& Lovegrove, P.J. (2011). How teenage fathers matter for children: evidence from the ECLS-B. Journal of Family Issues, 32, 3-30.

- Nascimento, P. (1999). "Ser homem ou nada": diversidade de experiências e estratégias de atualização do modelo hegemônico da masculinidade em Camaragibe/ PE. Dissertação de Mestrado, Universidade Federal de Pernambuco, Recife, Brasil.
- Sarti, C. A. (2005a). Família e individualidade: um problema moderno. In M. C. B. Carvalho (Ed.), A família contemporânea em debate (pp. 39-49). São Paulo: EDUC/Cortez.

- Sarti, C. A. (2005b). A família como espelho: um estudo sobre a moral dos pobres. 3. ed., São Paulo: Cortez Editora.

- Scavone, L. (2004). Dar a vida e cuidar da vida: feminismo e ciências sociais. São Paulo: Editora da UNESP.

- Scott, P. (2010). Homens, domesticidade e políticas públicas na saúde Reprodutiva. In B. Medrado, J. Lyra, M. Azevedo, \& J. Brasilino (Eds.), Homens e masculinidades: práticas de intimidade e políticas públicas. Recife: Instituto PAPAI.

- Suárez, M. et al. (1999). Violência, sexualidade e saúde reprodutiva. In L. Galvão \& J. Díaz (Eds.). Saúde sexual e reprodutiva no Brasil (pp.277-309). São Paulo: Hucitec/ Population Council.

- Trivedi, D., Brooks, F., Bunn, F., \& Graham, M. (2009). Early fatherhood: a mapping of the evidence base relating to pregnancy prevention and parenting support. Health Education Research, 24, 999-1028.

- Villela, W. V. \& Arilha, M. (2003). Sexualidade, gênero e direitos reprodutivos. In E. Berquó (Ed.), Sexo e Vida: panorama da saúde reprodutiva no Brasil (pp. 95-150). Campinas - SP: Editora da UNICAMP.

- Zaluar, A. (1994). A máquina e a revolta: as organizações populares e o significado da pobreza. 2ed., São Paulo: Brasiliense. 